\title{
Multigrid and Runge-Kutta time stepping applied to the uniformly non-oscillatory scheme for conservation laws
}

\author{
J.W. van der BURG*, J.G.M. KUERTEN and P.J. ZANDBERGEN \\ Faculty of Applied Mathematics, University of Twente, P.O. Box 217, 7500 AE Enschede, The Netherlands \\ (*author for correspondence)
}

Received 4 April 1990; accepted in revised form 18 January 1991

\begin{abstract}
It is known that Harten's uniformly non-oscillatory scheme is a second-order accurate scheme for discretizing conservation laws. In this paper a multigrid technique and Runge-Kutta time stepping with frozen dissipation is applied to Harten's scheme in order to obtain the steady-state solution. It is shown that these techniques applied to Harten's scheme lead to a better convergence to the steady-state solution of a first-order conservation law than applied to Jameson's scheme.
\end{abstract}

\section{Introduction}

In Computational Fluid Dynamics (CFD) the development of second-order accurate numerical schemes, which produce high-quality solutions, and the application of acceleration techniques are of significant interest. In general, the accuracy of a scheme which is first-order accurate in space, such as Roe's scheme [12], does not suffice for practical applications, particularly for the simulation of viscous flows. However, the extra accuracy obtained with higher-order schemes is at the expense of an increased calculation time per grid point.

In reference [8], Jameson constructed a pseudo second-order scheme by adding artificial dissipation to a central flux difference. This makes his scheme second-order accurate in a smooth field, but only first-order accurate in the neighbourhood of shocks. In order to keep the amount of calculation time within reasonable limits, Jameson applied Runge-Kutta time stepping with frozen dissipation. Further acceleration of convergence was achieved by a multigrid technique. Recently, Harten $[6,7]$ introduced a new concept in the construction of high-order accurate schemes, namely essentially non-oscillatory interpolation (ENO). One of these ENO schemes is the uniformly non-oscillatory (UNO) scheme described in [6], which is second-order accurate in the entire flow field.

The present work will investigate the multigrid behaviour of the UNO scheme in comparison with Jameson's and Roe's scheme when a time-explicit Runge-Kutta scheme with frozen dissipation is applied. Important for a successful multigrid performance is the damping of all possible Fourier modes in the discrete solution. A Fourier analysis will be applied to investigate the optimal damping rate of all Fourier components of the error in the multigrid process.

The presentation starts in the next section with a description of the dissipation models, i.e. Roe's, Jameson's and Harten's dissipation models. In the third section Runge-Kutta time stepping with frozen dissipation is discussed. In the fourth section the multigrid procedure is explained. The chosen multigrid routine consists of full multigrid (FMG) and the full approximation scheme (FAS). In the fifth section the Fourier analysis is described. The paper concludes with a presentation of the numerical results presented for a four-stage Runge-Kutta scheme. 


\section{The numerical schemes}

Three-dimensional fluid flow can be modelled by a set of nonlinear conservation laws, e.g. conservation of mass, conservation of momentum and conservation of energy. The present investigation deals with a simplified model, namely a one-dimensional scalar conservation law, which is defined on a finite space domain. Together with an initial condition this is referred to as the initial boundary value problem or IBVP.

The scalar conservation law can be cast in the general form

$$
u_{t}+f(u)_{x}=0 \text { with }(x, t) \in \Omega,
$$

where $x$ denotes the space coordinate and $t$ the time. The domain $\Omega$ will be taken to be $[0,1] \times(0, \infty)$. The flux function $f$ may depend nonlinearly on $u=u(x, t)$ and is assumed to be convex. In the case $f(u)=\frac{1}{2} u^{2}$, equation (1) represents the inviscid Burgers' equation. Application of the chain rule to the spatial derivative in (1) results in the quasi-linear form

$$
u_{t}+a(u) u_{x}=0
$$

where the function $a(u)$ denotes the characteristic wave speed,

$$
a(u)=\frac{\mathrm{d} f}{\mathrm{~d} u}(u) .
$$

A characteristic is a curve in the $(x, t)$ plane,

$$
x=a(u) t+x_{0},
$$

along which the solution $u(x, t)$ is constant and therefore satisfies

$$
\frac{\mathrm{d} x}{\mathrm{~d} t}=a(u)
$$

Due to the finiteness of the domain, initial and boundary conditions are needed. The initial condition of the IBVP can be stated as

$$
u(x, 0)=\phi(x)
$$

where $\phi(x)$ is a given function on the interval $x \in[0,1]$.

A time-dependent boundary condition

$$
u(0, t)=b_{0}(t) \text { or } u(1, t)=b_{1}(t)
$$

is imposed dependent upon when the characteristic (4) is directed into the domain $\Omega$. The domain and the boundary conditions are shown in Fig. 1.

Numerical solution of the IBVP requires the discretization of the conservation law (1). Introduce the grid function $u_{j}=u\left(x_{j}\right)$ on the equidistant grid $x_{j}=j h$, where $j=0, \ldots, m$, and the mesh size is $h=1 / \mathrm{m}$. A cell is defined for the grid point $j=1, \ldots, m-1$ as the interval $\left[x_{j-\frac{1}{2}}, x_{j+\frac{1}{2}}\right]$, i.e. the line segment between the midpoints of two adjacent grid nodes. A part of the equidistant grid is shown in Fig. 2. 


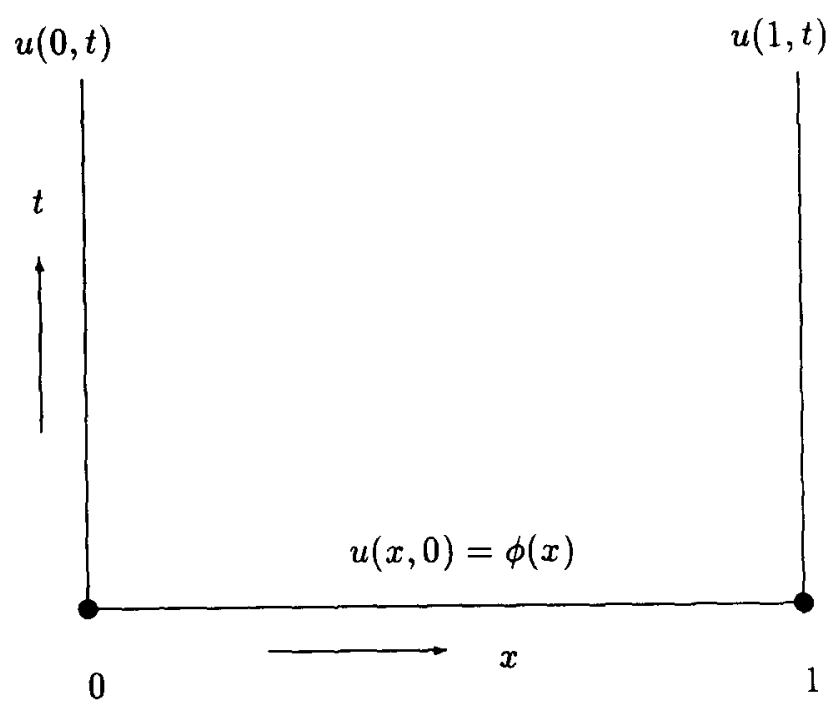

Fig. 1. The computational domain $\Omega$.

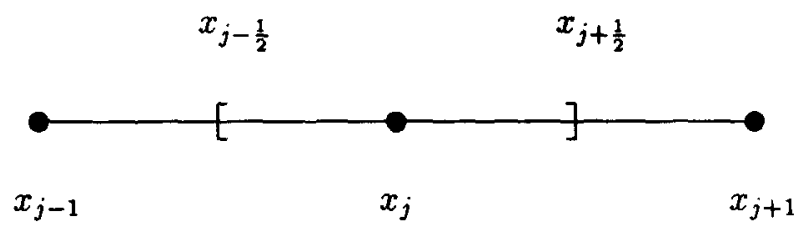

Fig. 2. The equidistant grid with mesh size $h$. The area between the brackets corresponds to a cell.

Taking a central difference for the spatial derivative, equation (1) has the semi-discrete relation

$$
\frac{\mathrm{d} u_{j}}{\mathrm{~d} t}+\frac{f_{j+\frac{1}{2}}-f_{j-\frac{1}{2}}}{h}=0, \quad j=1, \ldots, m-1,
$$

where $f_{j \pm \frac{1}{2}}$ represents the discrete flux at the cell faces $x=x_{j \pm \frac{1}{2}}$. The advantage of this formulation is that the discrete system remains conservative. The calculation of the discrete fluxes will be discussed in detail later on in this section.

The system of ordinary differential equations (8) can be solved by applying an explicit time-integration routine. In order to keep the integration stable a Courant-Friedrichs-Lewy (CFL) condition [2] has to be satisfied,

$$
\sigma \leq \sigma_{\max }
$$

where $\sigma$ is the CFL-number and $\sigma_{\max }$ is the maximum CFL number, which depends on the choice of the time-integration routine and the space discretization. The time step can then be calculated from

$$
\Delta t=\frac{h \sigma}{\max _{(j=0, \ldots, m-1)}\left|a_{j+\frac{1}{2}}\right|},
$$


where $a_{j+\frac{1}{2}}$ is an approximation of the characteristic wave-speed (3) at cell faces. For the sake of convenience the ratio of the time step and the mesh size is denoted by

$$
\lambda=\frac{\Delta t}{h} .
$$

A boundary can be an inlet or an outlet, depending on the sign of the characteristic wave speed. For an inflow boundary $\left(a_{\frac{1}{2}}>0\right.$ or $\left.a_{m-\frac{1}{2}}<0\right)$ the time-dependent condition (7) holds, yielding

$$
u_{j}^{n}=g\left(t_{n}\right)
$$

where $j=0$ or $j=m$. For an outflow boundary the solution can be extrapolated linearly, see for instance [8].

In the remainder of this section the three dissipation models investigated here, will be discussed. One of these methods (Roe's) is first-order accurate in space, whereas the other two are second-order accurate in space. The solution methods can all be put in the semi-discrete form (8). In each case the discrete flux appearing in that equation can be split into two parts, namely a convective flux and a dissipative (or diffusive) flux

$$
f_{j+\frac{1}{2}}=C_{j+\frac{1}{2}}-D_{j+\frac{1}{2}}, \quad j=0, \ldots, m-1,
$$

where the convective and dissipative parts can be expressed as

$$
C_{j+\frac{1}{2}}=\frac{1}{2}\left(f\left(u_{j}\right)+f\left(u_{j+1}\right)\right), \quad j=0, \ldots, m-1,
$$

and

$$
D_{j+\frac{1}{2}}=D_{j+\frac{1}{2}}\left(u_{0}, \ldots, u_{m}\right), j=0, \ldots, m-1
$$

respectively. The solution methods differ in the calculation of the dissipative flux $D_{j+\frac{1}{2}}$. Relation (8) is said to be consistent (see [3]) with the conservation law (1) if the dissipative flux satisfies

$$
D_{j+\frac{1}{2}}\left(u_{0}, \ldots, u_{m}\right)=\frac{1}{2}\left|a_{j+\frac{1}{2}}\right|\left(u_{j+1}-u_{j}\right)+\mathrm{O}\left(h^{2}\right), \quad D_{j+\frac{1}{2}}(c, \ldots, c)=0,
$$

for any constant $c$.

\section{Roe's dissipation model}

Roe's scheme is a first-order upwind scheme. This means in fact that the central flux difference in (8) is calculated by using the first-order accurate forward or backward difference, depending on the sign of the characteristic wave speed. The method is described in [12]. The dissipative flux equals

$$
D_{j+\frac{1}{2}}=\frac{1}{2} \psi\left(a_{j+\frac{1}{2}}\right) \Delta_{j+\frac{1}{2}} u,
$$


where $\Delta_{j+\frac{1}{2}} u=u_{j+1}-u_{j}$ and $\psi(a)=|a|$ is the dissipation coefficient. Due to the fact that the amount of numerical dissipation may become too low near a sonic point, where $a(u)$ equals zero, a correction of $\psi$ is needed which prevents entropy-violation [15]. This is

$$
\psi(a)= \begin{cases}|a| & \text { if }|a|>\delta, \\ \left(a^{2}+\delta^{2}\right) /(2 \delta) & \text { if }|a| \leqslant \delta .\end{cases}
$$

Here $\delta$ is a small parameter given by $\delta=\max \left\{0, a_{j+\frac{1}{2}}-a_{j}, a_{j+1}-a_{j+\frac{1}{2}}\right\}$ for $a=a_{j+\frac{1}{2}}$. The characteristic wave speed $a_{j+\frac{1}{2}}$ is defined for $j=0, \ldots, m-1$ by

$$
a_{j+\frac{1}{2}}= \begin{cases}\Delta_{j+\frac{1}{2}} f / \Delta_{j+\frac{1}{2}} u & \text { if } \Delta_{j+\frac{1}{2}} u \neq 0, \\ a_{j} \equiv a\left(u_{j}\right) & \text { if } \Delta_{j+\frac{1}{2}} u=0,\end{cases}
$$

where $a\left(u_{j}\right)$ is calculated with equation (3).

\section{Jameson's dissipation model}

The second method considered here is Jameson's scheme [8], which is a pseudo second-order accurate scheme. The idea of Jameson's scheme is to use a fourth-order difference term in the calculation of the central flux difference in (8). This provides a base level of dissipation throughout the domain, sufficient to prevent odd-even decoupling, but not sufficient to prevent oscillations in the neighbourhood of shock waves in the numerical solution. In order to capture a shock wave, an additional second-order difference term is added locally by a sensor designed to detect discontinuities. In the calculation of the discrete flux (13) the second- and fourth-order dissipation become first- and third-order differences, so that the dissipative flux can be written as

$$
D_{j+\frac{1}{2}}=\varepsilon_{j+\frac{1}{2}}^{(2)}\left|a_{j+\frac{1}{2}}\right| \Delta_{j+\frac{1}{2}} u-\varepsilon_{j+\frac{1}{2}}^{(4)}\left|a_{j+\frac{1}{2}}\right| \Delta_{j+\frac{1}{2}}^{3} u
$$

with $\Delta_{j+\frac{1}{2}}^{3} u=u_{j+2}-3 u_{j+1}+3 u_{j}-u_{j-1}$. In order to prevent entropy violation near a sonic point the absolute value of the characteristic wave speed is calculated by

$$
\left|a_{j+\frac{1}{2}}\right|=\frac{1}{2}\left[\left|a\left(u_{j}\right)\right|+\left|a\left(u_{j+1}\right)\right|\right], \quad j=0, \ldots, m-1 .
$$

The coefficients $\varepsilon^{(2)}$ and $\varepsilon^{(4)}$ are functions of the value of a shock-sensor $\nu_{j+\frac{1}{2}}$ given by

$$
\nu_{j+\frac{1}{2}}=2 \max \left\{\nu_{j+2}, \nu_{j+1}, \nu_{j}, \nu_{j-1}\right\}, \quad j=0, \ldots, m-1,
$$

where

$$
\nu_{j}=\frac{\left|u_{j+1}-2 u_{j}+u_{j-1}\right|}{\left|u_{j+1}\right|+2\left|u_{j}\right|+\left|u_{j-1}\right|}, \quad j=1, \ldots, m-1 .
$$

Thus the shock sensor is $\mathrm{O}\left(h^{2}\right)$ in a smooth field and $\mathrm{O}(1)$ near a shock. Jameson writes the adaptive coefficients as

$$
\varepsilon_{j+\frac{1}{2}}^{(2)}=\min \left(\frac{1}{2}, \kappa^{(2)} \nu_{j+\frac{1}{2}}\right), \quad \varepsilon_{j+\frac{1}{2}}^{(4)}=\max \left(0, \kappa^{(4)}-\alpha \nu_{j+\frac{1}{2}}\right)
$$


with $\kappa^{(2)}=1, \kappa^{(4)}=1 / 32$ and $\alpha=2$. Comparison of (16) and (19) shows that Jameson's scheme is generally less dissipative than Roe's scheme on a sufficiently fine grid.

The difference formula $\Delta_{j+\frac{1}{2}}^{3}$ is undefined at the boundaries. It is replaced by

$$
\Delta_{\frac{1}{2}}^{3}=u_{2}-2 u_{1}+u_{0}, \quad \Delta_{m-\frac{1}{2}}^{3}=-u_{m}+2 u_{m-1}-u_{m-2}
$$

and the shock sensor (21) is set equal to zero,

$$
\nu_{-1}=\nu_{0}=\nu_{m}=\nu_{m+1}=0
$$

\section{Harten's dissipation model}

Recently, Harten introduced the concept of ENO interpolation in order to create high-order accurate schemes. The second-order accurate ENO scheme described in [6], which Harten calls the UNO scheme, will be discussed below. The ENO schemes share many desirable properties with the total variation diminishing (TVD) schemes. The major difference between corresponding ENO schemes and TVD schemes is that the TVD schemes are one order less accurate at extrema of the numerical solution (see [6]).

The dissipative flux of the UNO scheme contains an extra term in comparison with Roe's dissipative flux (16). This makes the scheme less dissipative. It is

$$
D_{j+\frac{1}{2}}=D_{j+\frac{1}{2}}^{\mathrm{Roe}}- \begin{cases}\frac{1}{2} \frac{a_{j+\frac{1}{2}}\left(1-\lambda a_{j-\frac{1}{2}}\right)}{\left[1+\lambda\left(a_{j+\frac{1}{2}}-a_{j-\frac{1}{2}}\right)\right]} S_{j} & \text { if } a_{j+\frac{1}{2}} \geqslant 0, \\ -\frac{1}{2} \frac{a_{j+\frac{1}{2}}\left(1+\lambda a_{j+\frac{3}{2}}\right)}{\left[1+\lambda\left(a_{j+\frac{3}{2}}-a_{j+\frac{1}{2}}\right)\right]} S_{j+1} & \text { if } a_{j+\frac{1}{2}}<0,\end{cases}
$$

where $a_{j+\frac{1}{2}}$ is given by equation (18). The dissipative flux in the UNO scheme depends on the time step, as can be seen from the parameter $\lambda$ which is defined by (11). The reason for the presence of this parameter can be explained as follows.

Let $a_{j+\frac{1}{2}} \geqslant 0$ and assume that the characteristic wave speeds are distributed linearly between $x=x_{j-\frac{1}{2}}$ and $x=x_{j+\frac{1}{2}}$ at time $t=t_{n}$ (see also Fig. 3). The characteristic passing through the point $(x, t)=\left(x_{j+\frac{1}{2}}, t_{n+1}\right)$ can now be determined by applying a linear interpolation of the characteristic wave speeds. The case $a_{j+\frac{1}{2}} \leqslant 0$ is similar. The corresponding characteristic wave speed is second-order accurate in space and is equal to the ratio appearing in (24).

Apart from the ratio of the characteristic wave speeds, the UNO dissipation contains the term $S_{j}$, which constitutes an approximation of $h$ times the derivative $\partial u / \partial x$. In order to

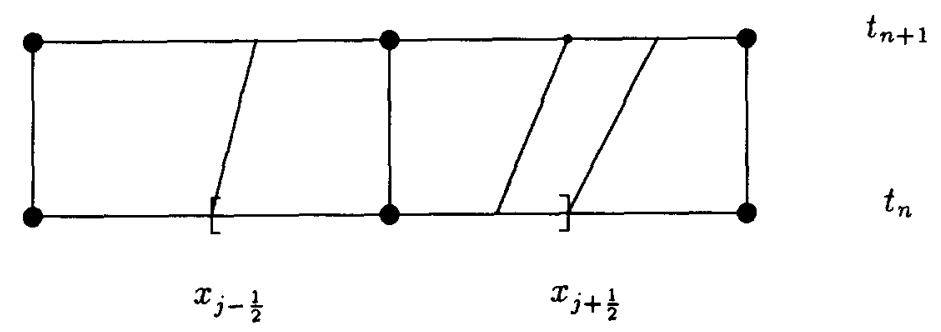

Fig. 3. Linear interpolation of the characteristics in cell $\left[x_{j-\frac{1}{2}}, x_{j+\frac{1}{2}}\right]$ in case $a_{j+\frac{1}{2}}>0$ in order to obtain the characteristic crossing the point $\left(x_{j+\frac{1}{2}}, t_{n+1}\right)$. 
retain second-order accuracy in the case of discontinuities in the solution, this approximation is determined in the following way.

The second difference of the solution at the grid nodes $x=x_{j}$ is defined as

$$
\Delta_{j}^{2} u=u_{j+1}-2 u_{j}+u_{j-1} .
$$

At boundaries this quantity is set equal to zero,

$$
\Delta_{0}^{2} u=0, \quad \Delta_{m}^{2} u=0
$$

In order to obtain a second difference in midpoints, the minmod limiter is applied,

$$
\Delta_{j+\frac{1}{2}}^{2} u=\operatorname{minmod}\left(\Delta_{j}^{2} u, \Delta_{j+1}^{2} u\right)
$$

where the limiter is defined as $\operatorname{minmod}(a, b)=\operatorname{median}(a, 0, b)$. As a consequence, $\Delta_{j+\frac{1}{2}}^{2} u$ becomes zero at an inflection point. The approximation $S_{j}$ of $h$ times the derivative $\partial u / \partial x$ can now be obtained from

$$
\begin{aligned}
& S_{j}^{+}=\Delta_{j+\frac{1}{2}} u-\frac{1}{2} \Delta_{j+\frac{1}{2}}^{2} u, \quad S_{j}^{-}=\Delta_{j-\frac{1}{2}} u+\frac{1}{2} \Delta_{j-\frac{1}{2}}^{2} u, \\
& S_{j}=\operatorname{minmmod}\left(S_{j}^{+}, S_{j}^{-}\right),
\end{aligned}
$$

where $S_{j}^{+}$and $S_{j}^{-}$are approximations of $h$ times the derivative $\partial u / \partial x$ in $x=x_{j+\frac{1}{2}}$ and $x=x_{j-\frac{1}{2}}$ respectively. This leads to the six possibilities for $S_{j}$ shown in Table 1 .

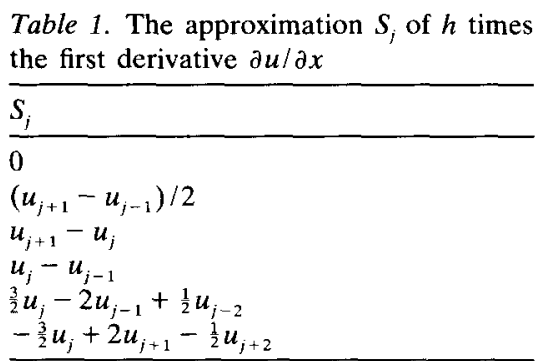

The scheme described here is second-order accurate in the entire flow field. The accuracy can even be increased by including higher-order differences in the dissipator.

\section{Runge-Kutta time stepping with frozen dissipation}

In order to compute an approximate steady-state solution of equation (8) with fixed boundary conditions, explicit Runge-Kutta time stepping with frozen dissipation can be applied to the system (8), when computations are carried out for a large number of time steps. The system (8) can more generally be written as

$$
\frac{\mathrm{d} \mathbf{u}}{\mathrm{d} t}+N(\mathbf{u})=\mathbf{g}
$$


where $\mathbf{u}=\left[u_{0}, \ldots, u_{m}\right]^{T}$ and $\mathbf{g}$ is a possible right-hand-side vector, which equals zero for (8).

Using the discrete flux (13), the operator $N$ can be put in the form

$$
N(\mathbf{u})=C(\mathbf{u})-D(\mathbf{u})
$$

where

$$
C_{j}(\mathbf{u})=\left(C_{j+\frac{1}{2}}-C_{j-\frac{1}{2}}\right) / h, \quad j=1, \ldots, m-1,
$$

and

$$
D_{j}(\mathbf{u})=\left(D_{j+\frac{1}{2}}-D_{j-\frac{1}{2}}\right) / h, \quad j=1, \ldots, m-1 .
$$

In order to explain the Runge-Kutta procedure applied to (29), the defect vector $\mathbf{d}$ is introduced as

$$
\mathbf{d}=N(\mathbf{u})-\mathbf{g} .
$$

The advancement of one time step $\Delta t$ with a time-explicit $k$-stage Runge-Kutta scheme can now be written as

$$
\begin{aligned}
& \mathbf{u}^{(0)}=\mathbf{u}(t), \\
& \mathbf{u}^{(1)}=\mathbf{u}^{(0)}-\alpha_{1} \Delta t \mathbf{d}^{(0)}, \\
& \mathbf{u}^{(2)}=\mathbf{u}^{(0)}-\alpha_{2} \Delta t \mathbf{d}^{(1)}, \\
& \cdots \\
& \mathbf{u}^{(k-1)}=\mathbf{u}^{(0)}-\alpha_{k-1} \Delta t \mathbf{d}^{(k-2)}, \\
& \mathbf{u}^{(k)}=\mathbf{u}^{(0)}-\alpha_{k} \Delta t \mathbf{d}^{(k-1)}, \\
& \mathbf{u}(t+\Delta t)=\mathbf{u}^{(k)},
\end{aligned}
$$

where $\alpha_{i}$ are constants and $\alpha_{k}=1$ for consistency. The coefficients $\alpha_{i}$ and the number of Runge-Kutta stage $k$ can easily be changed, which makes the Runge-Kutta routine a flexible time-step procedure.

In order to reduce the calculation time, Jameson freezes the dissipative fluxes (32) present in the operator $N$ in the Runge-Kutta stages $2, \ldots, k$. As a consequence the defect vectors become

$$
\begin{aligned}
& \mathbf{d}^{(0)}=C\left(\mathbf{u}^{(0)}\right)-D\left(\mathbf{u}^{(0)}\right)-\mathbf{g}, \\
& \mathbf{d}^{(k)}=C\left(\mathbf{u}^{(k)}\right)-\beta D\left(\mathbf{u}^{(1)}\right)-(1-\beta) D\left(\mathbf{u}^{(0)}\right)-\mathbf{g}, \quad k \geqslant 1,
\end{aligned}
$$

where $\beta$ is a constant satisfying $0 \leqslant \beta \leqslant 1$.

\section{Multigrid}

One of the pioneers of the multigrid technique is Brandt [1], who originally applied the concept to elliptic partial differential equations. A general review of the multigrid technique is presented in the work of Stuben and Trottenberg [14].

The multigrid technique for numerically solving hyperbolic partial differential equations is less well developed. Spekreijse [13] solved the Euler equations for inviscid flow using a multigrid procedure. Koren [9] extended the work of Spekreijse to the Navier-Stokes 
equations. Both Spekreijse and Koren used Newton iteration as a relaxation technique for a multigrid procedure. The disadvantage of Newton iteration is that it requires the convective and the dissipative fluxes to be continuously differentiable. Dick [4] numerically solved the steady Euler equations using symmetric Gauss-Seidel as a relaxation technique for a multigrid procedure. A requirement for this technique was that the system had to be positive. Both requirements are not necessary for Runge-Kutta time stepping.

Lallemand and Koren [10] showed for the van Leer scheme that Runge-Kutta time stepping is an effective relaxation procedure for a multigrid technique. The application of the Runge-Kutta technique (34) in a single-grid calculation with a small mesh size $h$ requires an intolerably high number of iterations. This is due to the fact that the Runge-Kutta time stepping is not able to fastly damp the low Fourier modes present in the solution. In a multigrid technique these modes are damped by using coarser grids.

The multigrid technique in the present work can only be applied to steady-state calculations. Consider the following more general equation in order to explain the multigrid routine used here:

$$
N(u)=g(u)
$$

where $N$ represents the nonlinear operator

$$
N(u)=a(u) \frac{\partial u}{\partial x}
$$

and $a$ and $g$ are known functions. The discretization $N^{h}$ of the nonlinear operator $N$ on an equidistant grid with mesh size $h$ results in a system of discrete equations

$$
N^{h}\left(\mathbf{u}^{h}\right)=\mathbf{g}^{h},
$$

where the boundary conditions are included. The addition of a time derivative to system (38) results in equation (29), which can be solved with the Runge-Kutta time stepping technique (34). The steady-state solution of (29) also satisfies (38).

Application of a number of Runge-Kutta time steps to a specified initial condition results in an approximation $\tilde{\mathbf{u}}^{h}$. The algebraic error is defined as the difference between the steady-state solution $\mathbf{u}^{h}$ and the approximation $\tilde{\mathbf{u}}^{h}$ to this solution,

$$
\mathbf{v}^{h}=\mathbf{u}^{h}-\tilde{\mathbf{u}}^{h}
$$

An appropriate measure for the convergence forms the $L_{1}$-norm of the defect vector (33) evaluated at the approximated solution $\tilde{\mathbf{u}}^{h}$,

$$
\mathbf{d}^{h}=N^{h}\left(\tilde{\mathbf{u}}^{h}\right)-\mathbf{g}^{h} .
$$

This defect vector is zero when the steady-state solution $\mathbf{u}^{h}$ is substituted in (40).

The basic feature in the multigrid technique is the V-cycle. The following text will explain every step of the V-cycle by assuming only two grid levels. The extension to more than two grid levels is straightforward. The V-cycle starts at the fine grid with mesh size $h$, where $\mu_{1}$ time steps or pre-relaxations are performed. The obtained solution will then be used to start 
the time stepping at the coarse grid with mesh size $H=2 h$. In order to use the Runge-Kutta technique at the coarse grid, a so-called coarse-grid equation of the form (38) is needed. This equation can be derived as follows. Substitute the right-hand-side vector of (38) in the defect vector (40). This yields

$$
\mathbf{d}^{h}=N^{h}\left(\tilde{\mathbf{u}}^{h}\right)-N^{h}\left(\tilde{\mathbf{u}}^{h}+\mathbf{v}^{h}\right),
$$

where (39) is used. The coarse-grid equations form an approximation of (41) at a grid with mesh size $H=2 h$,

$$
\mathbf{d}^{H}=N^{H}\left(\tilde{\mathbf{u}}^{H}\right)-N^{H}\left(\tilde{\mathbf{u}}^{H}+\hat{\mathbf{v}}^{H}\right) .
$$

This can be reformulated as

$$
N^{H}\left(\tilde{\mathbf{w}}^{H}\right)=\mathbf{g}^{H},
$$

where

$$
\tilde{\mathbf{w}}^{H}=\tilde{\mathbf{u}}^{H}+\hat{\mathbf{v}}^{H} \quad \text { and } \quad \mathbf{g}^{H}=N^{H}\left(\tilde{\mathbf{u}}^{H}\right)-\mathbf{d}^{H} .
$$

Equation (43) has to be solved for $\tilde{\mathbf{w}}^{h}$. In order to start the Runge-Kutta time stepping at the coarse grid, the solution vector $\tilde{\mathbf{u}}^{H}$ and the defect vector $\mathbf{d}^{H}$ are needed. These vectors can be found by applying a restriction operator $I_{h}^{H}$,

$$
\tilde{\mathbf{u}}^{H}=I_{h}^{H} \tilde{\mathbf{u}}^{h}, \quad \mathbf{d}^{H}=I_{h}^{H} \mathbf{d}^{h} .
$$

For the restriction operator $I_{h}^{H}$ full weighting is taken with injection at the boundaries, i.e.

$$
\begin{aligned}
& u_{j}^{H}=\frac{1}{4} u_{2 j-1}^{h}+\frac{1}{2} u_{2 j}^{h}+\frac{1}{4} u_{2 j+1}^{h}, \quad j=1, \ldots, m / 2-1, \\
& u_{0}^{H}=u_{0}^{h}, \quad u_{m / 2}^{H}=u_{m}^{h} .
\end{aligned}
$$

Next, $\mu_{0}$ time steps are performed at the coarse grid where the right-hand-side vector $\mathbf{g}^{H}$ is frozen. This results in an approximation for $\tilde{\mathbf{w}}^{H}$.

The next stage of the V-cycle consists of the prolongation of the correction

$$
\hat{\mathbf{v}}^{H}=\tilde{\mathbf{w}}^{H}-I_{h}^{H} \tilde{\mathbf{u}}^{h}
$$

to the fine grid. This correction denotes the difference between the final solution and the initial solution at the coarse grid. The transfer of the correction to the fine grid is done by means of the interpolation operator $I_{H}^{h}$. For the interpolation operator $I_{H}^{h}$ linear interpolation is used

$$
u_{2 j}^{h}=u_{j}^{H}, \quad u_{2 j+1}^{h}=\frac{1}{2}\left(u_{j}^{H}+u_{j+1}^{H}\right), \quad j=0, \ldots, m / 2 .
$$


The new fine-grid solution is found by adding the interpolated correction to the already known fine-grid solution,

$$
\tilde{\mathbf{u}}^{h} \Leftarrow \tilde{\mathbf{u}}^{h}+I_{H}^{h} \hat{\mathbf{v}}^{H} \text {. }
$$

With this fine-grid solution, $\mu_{2}$ time steps or post-relaxations are performed. Again the right-hand-side vector of the fine-grid equation is kept frozen during the time stepping.

The V-cycle can easily be extended to more than two grid levels (see Fig. 4). Starting from a fine grid, the coarsest grid can be reached by subsequently performing $\mu_{1}$ pre-relaxations followed by a restriction. At the coarsest grid, $\mu_{0}$ relaxations are taken. Next, the maximum grid level can be reached by subsequently performing a prolongation followed by $\mu_{2}$ post-relaxations.

The multigrid technique in the present work consists of two parts. In the first part full multigrid (FMG) is employed in order to obtain an accurate initial solution vector at the finest grid. In the second part the full approximation scheme (FAS) is used until the solution has converged.

The FMG stage starts at the coarsest grid level 1 by performing $\mu_{0}$ relaxations with $\mathbf{g}^{H}=0$. Next, the solution is transferred to grid level 2 with the interpolation operator $I_{H}^{h}$. The right-hand-side vector at the finer grid is set to $\mathbf{g}^{h}=\mathbf{0}$. One V-cycle is applied resulting in a better solution at grid level 2. Next, the transfer operator $I_{H}^{h}$ gives an initial solution at grid level 3. This procedure is repeated until the maximum number of grid levels is reached. The structure of the FMG algorithm is called the F-cycle. The initial stages of the F-cycle are shown in Fig. 5.

The FAS stage starts with an approximation of the solution at the finest grid level obtained by employing full multigrid. A previously set number of V-cycles can now be performed until the solution at the finest grid is converged to the steady-state solution. Characteristic for the FAS stage is that the high-frequency Fourier modes as well as the low-frequency Fourier modes are damped sufficiently. The high-frequency modes are damped by the relaxations at the finest grid, whereas the low-frequency modes are damped by the multigrid process.

The computational cost of one V-cycle with $\mu_{1}$ pre-relaxations, $\mu_{2}$ post-relaxations, $\mu_{0}$ relaxations at the coarsest grid and $l$ grid levels can be expressed in terms of the work $W U$ needed to perform one time step at the finest grid. In case the work needed for employing

Level

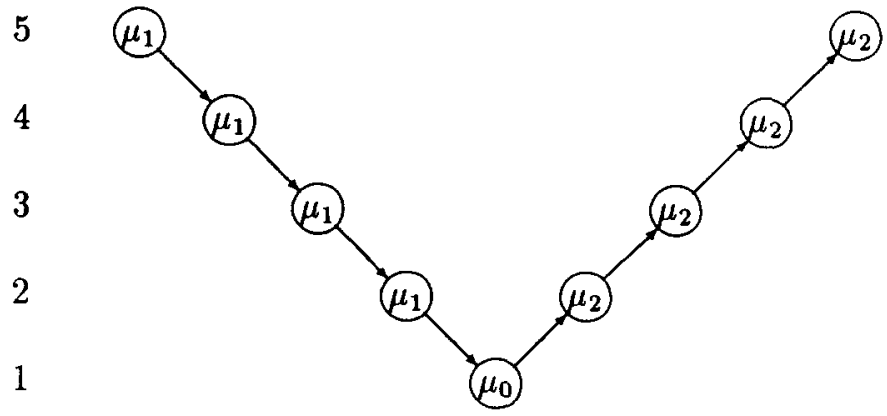

Fig. 4. One V-cycle with 5 grid levels. 


\section{Level}

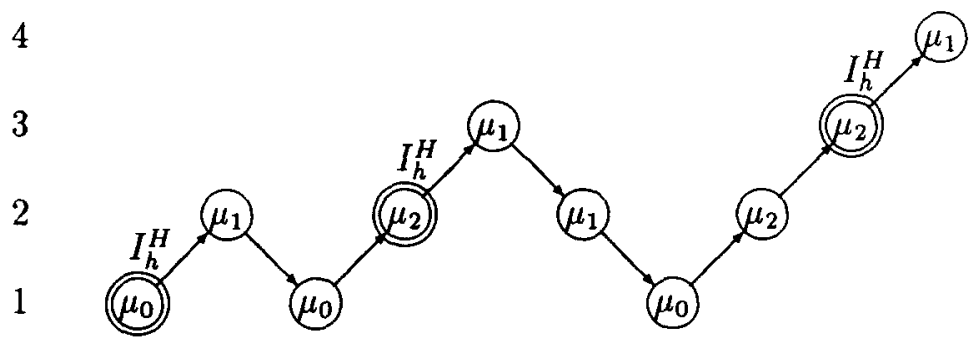

Fig. 5. The initial procedure of the full multigrid stage.

the transfer operators is neglected this is

$$
W U V(l)=\left[\left(\mu_{1}+\mu_{2}\right) \sum_{i=1}^{l-1} 2^{1-i}+\mu_{0} 2^{1-l}\right] \times W U,
$$

which is approximately

$$
W U V(l) \simeq 2\left(\mu_{1}+\mu_{2}\right)
$$

for large values of $l$.

Similarly the computational cost of the initial F-cycle can be calculated. The amount of work for one F-cycle is

$$
W U F(l)=\mu_{0} 2^{1-l} \times W U+W U V(l) \sum_{i=1}^{l-1} 2^{1-i},
$$

which is approximately

$$
W U F(l) \simeq 4\left(\mu_{1}+\mu_{2}\right)
$$

for large values of $l$.

\section{Fourier analysis of error reduction}

In this section a multi-level Fourier analysis will be applied to the linearized schemes in order to examine the performance of the combination of the multigrid technique and Runge-Kutta time stepping with the three different dissipation models. For a given scheme (8) the analysis results in an upper bound for the amplification of the Fourier modes in a multigrid V-cycle. This linear result is expected to be an upper bound for the error reduction obtained in a numerical calculation with the nonlinear inviscid Burgers' equation.

The linear form of a scheme (8) is obtained by considering the linear flux function $f(u)=u$. The consequence of this is that the characteristic wave speed is constant, i.e.

$$
a_{j+\frac{1}{2}}=a=1, \quad j=0, \ldots, m-1 .
$$


The linearization leads to the following expressions for the convective fluxes (14):

$$
C_{j+\frac{1}{2}}=\frac{1}{2}\left(u_{j}+u_{j+1}\right),
$$

and for the dissipative fluxes (see (15)):

$D_{j+\frac{1}{2}}^{\mathrm{Roe}}=\frac{1}{2} \Delta_{j+\frac{1}{2}} u$,

$D_{j+\frac{1}{2}}^{J \text { ameson }}=\tilde{\varepsilon}^{(2)} \Delta_{j+\frac{1}{2}} u-\tilde{\varepsilon}^{(4)} \Delta_{j+\frac{1}{2}}^{3} u$,

$D_{j+\frac{1}{2}}^{\text {Harten }}=D_{j+\frac{1}{2}}^{\text {Roe }}-\frac{1}{2}(1-\sigma) S_{j}$

where the nonlinear adaptive constants of Jameson's scheme are replaced by the constants $\tilde{\varepsilon}^{(2)}$ and $\tilde{\varepsilon}^{(4)}$. The boundary conditions are assumed to be periodic.

Consider the Fourier mode at time level $n$ with amplitude $\hat{u}_{\theta}^{h, n}$ and wave-number $\theta \in(0,2 \pi)$ on a grid with mesh size $h=1 / m$,

$$
u_{j}^{n}=\hat{u}_{\theta}^{h, n} \mathrm{e}^{\mathrm{i} \theta x_{j}}, \quad j=0, \ldots, m,
$$

where $\mathrm{i}=\sqrt{-1}$ and $x_{j}=j h$. A grid with mesh size $h$ containing $m+1$ equidistant mesh points can only represent $m$ Fourier modes, namely the modes with wave-number $\theta_{k}=2 \pi k$ for $k=1, \ldots, m$. Substitution of the Fourier mode with wave-number $\theta$ in equation (34), where equations $(30)-(33)$ and $(52,53)$ are used, yields the amplitude $\hat{u}_{\theta}^{h, n+1}$ after one RungeKutta time step.

The influence of the transfer operators during a V-cycle on the amplitudes of the Fourier modes is obtained by applying discrete Fourier transformations as in [5]. Define the discrete Fourier transformation at a certain grid with mesh size $h$ as

$$
u_{j}^{h}=h \sum_{k=1}^{m} \hat{u}_{\theta_{k}}^{h} \mathrm{e}^{\mathrm{i} \theta_{k} x_{j}}, \quad j=0, \ldots, m .
$$

Similarly, at the next coarser grid the discrete Fourier transform is defined as

$$
u_{j}^{H}=H \sum_{k=1}^{m / 2} \hat{u}_{\theta_{k}}^{H} \mathrm{e}^{\mathrm{i} \theta_{k} y_{j}}, j=0, \ldots, m / 2,
$$

where $y_{j}=j H$. The restriction operator (46) and the interpolation operator (48) couple the wave-numbers pairwise,

$$
\tilde{u}_{\theta_{k}}^{h}=\left[\begin{array}{c}
\hat{u}_{\theta_{k}}^{h} \\
\hat{u}_{\theta_{k+m} / 2}^{h}
\end{array}\right], \quad k=1, \ldots, m / 2 .
$$

The restriction operator yields the following amplitude of the Fourier mode with wavenumber $\theta_{k}$,

$$
\hat{u}_{\theta_{k}}^{H}=\left[\frac{1}{2}+\frac{1}{2} \cos \left(\theta_{k} h\right), \frac{1}{2}-\frac{1}{2} \cos \left(\theta_{k} h\right)\right] \tilde{u}_{\theta_{k}}^{h}, \quad k=1, \ldots, m / 2,
$$


and the interpolation operator gives

$$
\tilde{u}_{\theta_{k}}^{h}=\left[\begin{array}{c}
\frac{1}{2}+\frac{1}{2} \cos \left(\theta_{k} h\right) \\
\frac{1}{2}-\frac{1}{2} \cos \left(\theta_{k} h\right)
\end{array}\right] \hat{u}_{\theta_{k}}^{H}, \quad k=1, \ldots, m / 2 .
$$

Starting with the initial amplitudes $\hat{u}_{\theta_{k}}^{h}$ at the finest grid, one entire V-cycle can be performed resulting in the amplitudes $\hat{v}_{\theta_{k}}^{h}$ by applying the amplification rates obtained from RungeKutta time stepping, the restriction (58) and the interpolation (59). An upper bound for the amplification factor of the amplitudes in one V-cycle can now be found by taking

$$
A\left(\mu_{0}, \mu_{1}, \mu_{2}, \sigma\right)=\max _{(k=1, \ldots, m)}\left|\frac{\hat{v}_{\theta_{k}}^{h}}{\hat{u}_{\theta_{k}}^{h}}\right| .
$$

By varying the number of relaxations $\mu_{0}, \mu_{1}, \mu_{2}$ and the CFL-number, the amplification factor can be optimized.

In the linear case the amplification (60) will be accurate in case the initial solution contains the Fourier mode which results in the maximum value. The amplification rate $(60)$ is also expected to be an upper bound for the error reduction in the nonlinear case. However, in the nonlinear case higher amplification rates may be found due to the fact that the Fourier modes are mapped onto other modes during a relaxation on a single grid.

\section{Numerical results}

This section contains the numerical results obtained by applying multigrid and a four-stage Runge-Kutta scheme (34) with frozen dissipation. The numerical results for each dissipation model will be discussed separately.

The numerical calculation in this section is performed with the inviscid Burgers' equation. The discrete initial condition used in the calculation is

$$
\phi\left(x_{j}\right)=\cos \left(\pi x_{j}\right), \quad j=0, \ldots, m,
$$

where $x_{j}=j h$ and the boundary conditions are

$$
u_{0}^{n}=1, \quad u_{m}^{n}=-1,
$$

so that no outflow conditions are necessary in this calculation. At time $t=1 / \pi$ a shock develops in the solution, which is located at $x=1 / 2$ for large time. The steady-state solution is

$$
u_{j}=\left\{\begin{array}{ll}
1, & 0 \leqslant x_{j}<\frac{1}{2}, \\
0, & x_{j}=\frac{1}{2}, \\
-1, & \frac{1}{2}<x_{j} \leqslant 1,
\end{array} \quad \text { with } x_{j}=j h, j=0, \ldots, m\right.
$$

In order to reach the steady-state solution, multigrid is applied with a four-stage $(k=4)$ Runge-Kutta scheme with coefficients 
$\alpha_{1}=\frac{1}{4}, \quad \alpha_{2}=\frac{1}{3}, \quad \alpha_{3}=\frac{1}{2}, \quad \beta=1$

The dissipative flux is evaluated twice in each Runge-Kutta time step [8]. In the multigrid routine the coarsest grid contains 6 grid points. In the numerical calculation as well as in the Fourier analysis 7 grid levels have been used, which results in 321 grid points at the finest grid.

For this simple model problem the three dissipation models give the same steady-state solution. Hence, in this section only the convergence rate will be studied. For each dissipation model and in both the Fourier analysis and the numerical calculation the amplification factor per V-cycle will be optimized with respect to the CFL-number for a given choice of the number of relaxations at the coarsest grid, $\mu_{0}$, the number of pre-relaxations, $\mu_{1}$, and the number of post-relaxations, $\mu_{2}$. Afterwards, the optimal choice for the numbers of relaxations is determined, resulting in the optimal amplification rate $A_{\text {opt }}$.

In the Fourier analysis the upper bound for the amplification factor per V-cycle (60) is determined by choosing the initial amplitude vector as

$$
\hat{u}_{\theta_{k}}^{h, 0}= \begin{cases}1, & k=1, \ldots, m / 2, \\ 0, & k=m / 2+1, \ldots, m,\end{cases}
$$

so that the initial solution possesses only low-frequency Fourier components, which corresponds to the solution after the FMG stage.

In the numerical calculation the amplification factor per V-cycle is determined as follows. After the FMG stage and after the FAS stage the $L_{1}$-norm of the defect vector of the fine grid solution is calculated. The amplification factor in the FAS stage follows by taking the ratio of both $L_{1}$-norms.

In the discussion on the results of the numerical calculation, convergence plots will be used. A convergence plot shows the $L_{1}$-norm of the defect vector at the finest grid as a function of the number of work-units. The first point in the convergence plot is not of particular interest, because it is obtained by taking the $L_{1}$-norm of the defect vector of the initial solution at the coarsest grid. The second point is measured at the starting point of the FAS stage. The subsequent points are measured after each V-cycle in the FAS stage.

In the following the results for the dissipation models of Roe, Jameson and Harten will be discussed.

\section{Roe's dissipation model}

Table 2 shows the amplification rates per V-cycle and per work-unit obtained from the Fourier analysis for varying numbers of relaxations. The minimum and maximum allowable CFL-numbers are 0.0 and 2.0. The optimal value for the amplification factor per work-unit $A_{\text {opt }}=0.659$ is found for the parameters

$$
\mu_{0}=1, \quad \mu_{1}=1, \quad \mu_{2}=1, \quad \sigma=1.64 .
$$

In the numerical calculation the amplification rates shown in Table 3 are obtained. The optimum value for the amplification per work-unit $A_{\text {opt }}=0.556$ is found for the parameters 
Table 2. The amplification factor $A$ per V-cycle and per work-unit with respect to the CFL-number $\sigma$ as function of the relaxations $\mu_{0}, \mu_{1}, \mu_{2}$, obtained by applying the Fourier analysis to Roe's dissipation model

\begin{tabular}{llllll}
\hline$\mu_{0}$ & $\mu_{1}$ & $\mu_{2}$ & $\sigma$ & $A$ per V-cycle & $A$ per work-unit \\
\hline 0 & 1 & 1 & 1.80 & 0.433 & 0.811 \\
1 & 1 & 1 & 1.64 & 0.189 & 0.659 \\
2 & 1 & 1 & 1.64 & 0.189 & 0.659 \\
1 & 0 & 1 & 1.80 & 0.594 & 0.771 \\
1 & 1 & 1 & 1.64 & 0.189 & 0.659 \\
1 & 2 & 1 & 1.70 & 0.133 & 0.714 \\
1 & 1 & 0 & 1.68 & 0.478 & 0.691 \\
1 & 1 & 1 & 1.64 & 0.189 & 0.659 \\
1 & 1 & 2 & 1.70 & 0.133 & 0.714 \\
\hline
\end{tabular}

Table 3. The amplification factor $A$ per V-cycle and per work-unit with respect to the CFL-number $\sigma$ as function of the relaxations $\mu_{0}, \mu_{1}, \mu_{2}$, obtained from the numerical calculation with Roe's dissipation model

\begin{tabular}{llllll}
\hline$\mu_{0}$ & $\mu_{1}$ & $\mu_{2}$ & $\sigma$ & $A$ per V-cycle & $A$ per work-unit \\
\hline 0 & 1 & 1 & 1.60 & 0.096 & 0.557 \\
1 & 1 & 1 & 1.60 & 0.096 & 0.556 \\
2 & 1 & 1 & 1.60 & 0.096 & 0.557 \\
1 & 0 & 1 & 1.61 & 0.357 & 0.597 \\
1 & 1 & 1 & 1.60 & 0.096 & 0.556 \\
1 & 2 & 1 & 1.64 & 0.047 & 0.600 \\
1 & 1 & 1 & 1.61 & 0.299 & 0.547 \\
1 & 1 & 1 & 1.60 & 0.096 & 0.556 \\
1 & 1 & 2 & 1.65 & 0.051 & 0.610 \\
\hline
\end{tabular}

$$
\mu_{0}=1, \quad \mu_{1}=1, \quad \mu_{2}=1, \quad \sigma=1.60
$$

The convergence plot of the numerical calculation is shown in Fig. 6 . The final solution is reached in approximately 4.9 seconds at a VAX station 3100 . The difference between the numerical and analytical result is that the optimal amplification rate in the numerical calculation is significantly smaller than the upper bound found in the Fourier analysis. The

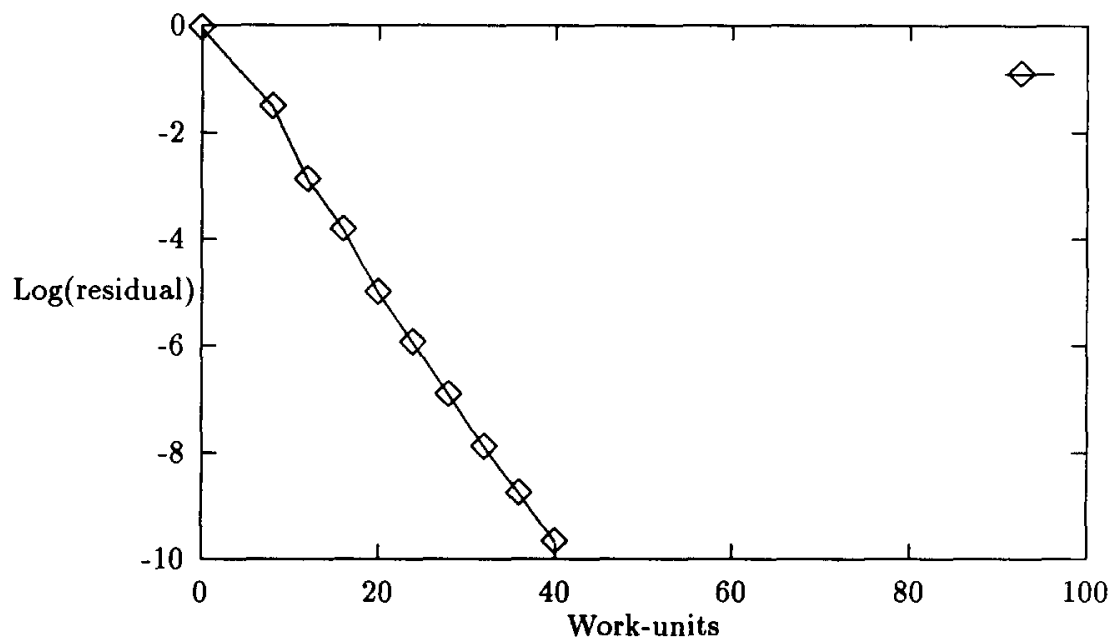

Fig. 6. The $L_{1}$-norm of the defect vector at the finest grid as a function of the number of work-units for Roe's dissipation model with the parameters $\mu_{0}=1, \mu_{1}=1, \mu_{2}=1$ and $\sigma=1.60$. 
reason for this is that a better damping is achieved due to the nonlinear form of Roe's dissipation model.

For the rest the numerical and analytical results correspond quite well. The optimal relaxation parameters are equal, whereas the optimal CFL-numbers are approximately the same.

\section{Jameson's dissipation model}

The dissipation in Jameson's model is essentially nonlinear. The fourth-order dissipation is present in the smooth parts of the solution, whereas the second-order dissipation is only locally present near shocks. Therefore, linearization of the equations is questionable for this model problem, where a strong shock is present. Since the solution is smooth in the main part of the domain, the optimal choice for the coefficients $\tilde{\varepsilon}^{(2)}$ and $\tilde{\varepsilon}^{(4)}$ in the linearized equations seems to be

$$
\tilde{\varepsilon}^{(2)}=0, \quad \tilde{\varepsilon}^{(4)}=\frac{1}{32} .
$$

The Fourier analysis results in the amplification rates shown in Table 4. The optimal amplification factor per work-unit $A_{\mathrm{opt}}=\mathbf{0 . 6 2 5}$ is found for the parameters

$$
\mu_{0}=2, \quad \mu_{1}=1, \quad \mu_{2}=0, \quad \sigma=2.44 .
$$

The minimum and maximum allowable CFL-numbers are approximately 1.5 and 2.7 . The reason for the existence of a lower bound is that in one pre-relaxation Jameson's scheme is not able to damp the Fourier components with a wave number near $\frac{1}{2} \pi$ at the fine grid sufficiently. The consequence of this is that the right-hand-side function becomes large at the next coarser grid and thus that the amplitudes blow up when taking relaxations at the coarse grid.

In the numerical calculation with the inviscid Burgers' equation the amplification rates shown in Table 5 are obtained. The optimal value for the amplification per work-unit $A_{\text {opt }}=0.765$ is found for

$$
\mu_{0}=2, \quad \mu_{1}=2, \quad \mu_{2}=1, \quad \sigma=2.03 .
$$

\begin{tabular}{|c|c|c|c|c|c|}
\hline$\mu_{0}$ & $\mu_{1}$ & $\mu_{2}$ & $\sigma$ & $A$ per $V$-cycle & $A$ per work-unit \\
\hline 0 & 1 & 0 & 2.71 & 0.930 & 0.964 \\
\hline 1 & 1 & 0 & 2.47 & 0.404 & 0.636 \\
\hline 2 & 1 & 0 & 2.44 & 0.391 & 0.625 \\
\hline 3 & 1 & 0 & 2.44 & 0.391 & 0.625 \\
\hline 4 & 1 & 0 & 2.44 & 0.391 & 0.625 \\
\hline 2 & 1 & 0 & 2.44 & 0.391 & 0.625 \\
\hline 2 & 2 & 0 & 2.35 & 0.168 & 0.640 \\
\hline 2 & 3 & 0 & 2.47 & 0.114 & 0.696 \\
\hline 2 & 1 & 0 & 2.44 & 0.391 & 0.625 \\
\hline 2 & 1 & 1 & 2.38 & 0.173 & 0.645 \\
\hline 2 & 1 & 2 & 2.47 & 0.115 & 0.697 \\
\hline 2 & 1 & 3 & 2.47 & 0.110 & 0.759 \\
\hline
\end{tabular}

Table 4. The amplification factor $A$ per V-cycle and per work-unit with respect to the CFL-number $\sigma$ as function of the relaxations $\mu_{0}, \mu_{1}, \mu_{2}$, obtained by applying the Fourier analysis to Jameson's dissipation model 
Table 5. The amplification factor $A$ per V-cycle and per work-unit with respect to the CFL-number $\sigma$ as function of the relaxations $\mu_{0}, \mu_{1}, \mu_{2}$, obtained from the numerical calculation with Jameson's dissipation model

\begin{tabular}{llllll}
\hline$\mu_{0}$ & $\mu_{1}$ & $\mu_{2}$ & $\sigma$ & $A$ per V-cycle & $A$ per work-unit \\
\hline 1 & 2 & 1 & 2.02 & 0.213 & 0.773 \\
2 & 2 & 1 & 2.03 & 0.201 & 0.765 \\
3 & 2 & 1 & 2.02 & 0.209 & 0.770 \\
2 & 1 & 1 & 2.13 & 0.512 & 0.846 \\
2 & 2 & 1 & 2.03 & 0.201 & 0.765 \\
2 & 3 & 1 & 2.03 & 0.170 & 0.801 \\
2 & 2 & 0 & 2.04 & 0.375 & 0.782 \\
2 & 2 & 1 & 2.03 & 0.201 & 0.765 \\
2 & 2 & 2 & 2.02 & 0.190 & 0.812 \\
\hline
\end{tabular}

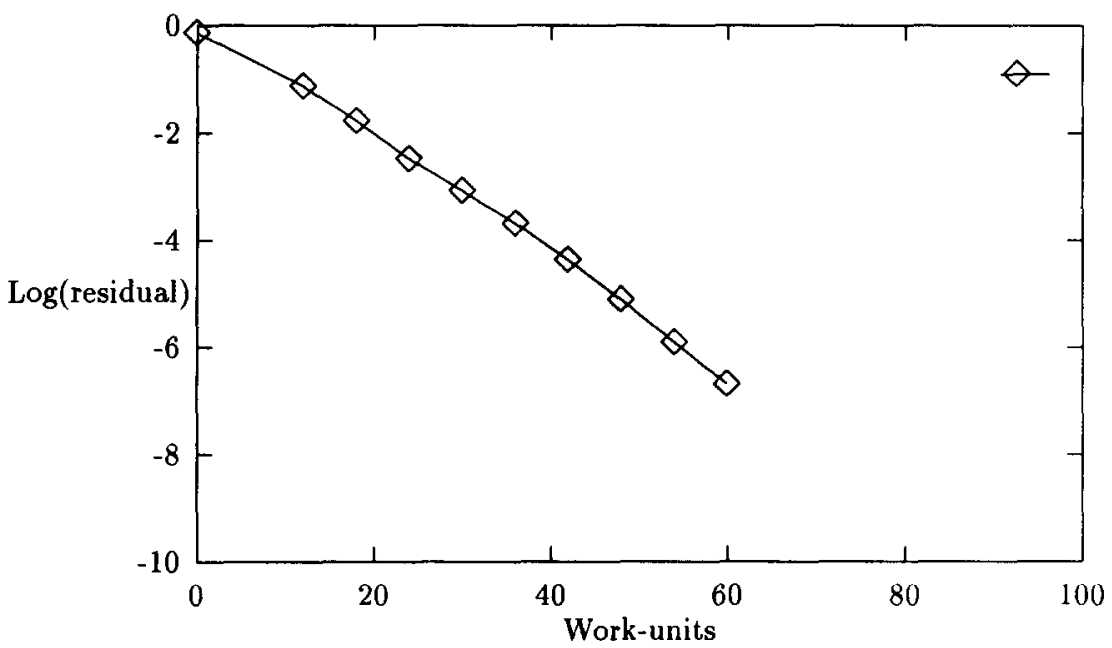

Fig. 7. The $L_{1}$-norm of the defect vector at the finest grid as a function of the number of work-units for Jameson's dissipation model with the parameters $\mu_{0}=2, \mu_{1}=2, \mu_{2}=1$ and $\sigma=2.03$.

The convergence plot of the numerical calculation is shown in Fig. 7. The final solution is reached in approximately 8.7 seconds on the same computer. The differences between the results of the Fourier analysis and the numerical calculation are significant. Firstly, one additional pre- and post-relaxation is needed in the optimization process of the numerical calculation. Secondly, the CFL-number in the calculation is smaller due to a higher amount of dissipation. Finally, the optimal amplification rate in the numerical calculation is larger than the upper bound found with the Fourier analysis. These differences are due to the fact that Jameson's dissipative flux is highly nonlinear.

\section{Harten's dissipation model}

A Fourier analysis cannot easily be applied to Harten's dissipation model, because this model is highly nonlinear due to the adaptivity of the difference stencils. In the smooth part of the solution the dissipation model mainly uses (see Table 1) the second-order central difference, the second-order forward difference and the second-order backward difference as approximation for $h$ times the derivative $\partial u / \partial x$.

Choosing the second-order central difference for $S_{j}$ one finds that the optimal amplification 
factor per work-unit $A_{\mathrm{opt}}=0.740$ is achieved with the parameters

$$
\mu_{0}=1, \quad \mu_{1}=1, \quad \mu_{2}=1, \quad \sigma=1.70 .
$$

The minimum and maximum allowable CFL-numbers are in this case 0.0 and 1.8 .

However, the choice of the second-order backward difference for $S_{j}$ restricts the stability interval. The optimal amplification factor per work-unit $A_{\mathrm{opt}}=0.754$ is then found for the parameters

$$
\mu_{0}=3, \quad \mu_{1}=1, \quad \mu_{2}=1, \quad \sigma=1.12,
$$

where the number of relaxations at the coarsest grid has been increased. The minimum and maximum allowable CFL-numbers are in this case 0.8 and 1.3.

In the numerical calculation the optimal value for the amplification factor per work-unit $A_{\text {opt }}=0.692$ is found for the parameters (see Table 6)

$$
\mu_{0}=0, \quad \mu_{1}=1, \quad \mu_{2}=1, \quad \sigma=1.20 .
$$

The convergence plot of the numerical calculation is shown in Fig. 8. The final solution is

Table 6. The amplification factor $A$ per V-cycle and per work-unit with respect to the CFL-number $\sigma$ as function of the relaxations $\mu_{0}, \mu_{1}, \mu_{2}$, obtained from the numerical calculation with Harten's dissipation model

\begin{tabular}{llllll}
\hline$\mu_{0}$ & $\mu_{1}$ & $\mu_{2}$ & $\sigma$ & $A$ per V-cycle & $A$ per work-unit \\
\hline 0 & 1 & 1 & 1.20 & 0.229 & 0.692 \\
1 & 1 & 1 & 1.20 & 0.230 & 0.692 \\
2 & 1 & 1 & 1.20 & 0.230 & 0.692 \\
0 & 0 & 1 & 1.28 & 0.534 & 0.731 \\
0 & 1 & 1 & 1.20 & 0.229 & 0.692 \\
0 & 2 & 1 & 1.20 & 0.116 & 0.698 \\
0 & 1 & 0 & 1.20 & 0.500 & 0.707 \\
0 & 1 & 1 & 1.20 & 0.229 & 0.692 \\
0 & 1 & 2 & 1.27 & 0.139 & 0.719 \\
\hline
\end{tabular}

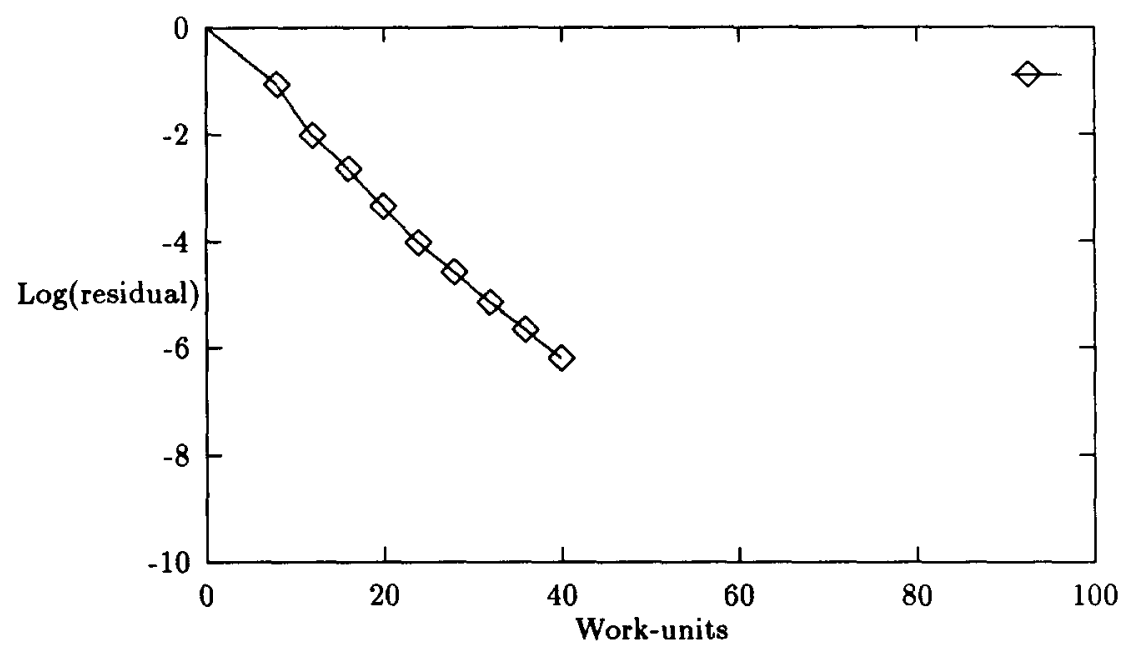

Fig. 8. The $L_{1}$-norm of the defect vector at the finest grid as a function of the number of work-units for Harten's dissipation model with the parameters $\mu_{0}=0, \mu_{1}=1, \mu_{2}=1$ and $\sigma=1.2$. 
reached in approximately 9.1 seconds on the same computer. The numerical amplification rate lies below the analytical upper bounds. The number of pre- and post-relaxations are equal in the numerical and anaytical case.

The first difference between the analytical and numerical results is the number of relaxations taken at the coarsest grid. In the numerical calculation this number is equal to zero, whereas in the Fourier analysis the optimal values are not equal to zero. Obviously, the second grid level containing 11 grid points is coarse enough for the Runge-Kutta procedure, so that all Fourier modes are damped sufficiently.

The second difference is the value of the CFL-number. The CFL-number compares with the CFL-number found for the second-order backward difference stencil, but is significantly smaller than the CFL-number found for the central difference stencil. In case a local minimum for the amplification rate for the central difference stencil is searched in the stability region $[0.8,1.3]$ of the second-order backward stencil, the Fourier analysis yields the amplification factor $A=0.858$ for the parameters

$$
\mu_{0}=1, \quad \mu_{1}=1, \quad \mu_{2}=1, \quad \sigma=1.20 \text {. }
$$

This CFL-number equals the CFL-number found in the numerical calculation. Besides, the numerical amplification rate lies well below the analytical upper bound.

\section{Conclusion}

Comparison of the numerical results of the discussed dissipation models shown in Figs 6-8 leads to the conclusion that the first-order Roe scheme performs best in combination with multigrid and Runge-Kutta time stepping. The method possesses the lowest amplification rate per V-cycle in comparison with Harten's and Jameson's methods. However, the first-order accurate Roe scheme is not very suited for viscous flow problems.

Among the second-order schemes, Harten's scheme shows the best amplification rate per $\mathrm{V}$-cycle. This is desirable, because Harten's dissipation model, which is really second-order accurate in space, is more expensive per V-cycle than Jameson's dissipation model, which is only first-order accurate at shocks. The calculation times necessary to reach a solution with an error of $10^{-6}$ are approximately the same for both schemes. It seems that the extension of Harten's dissipation model to multidimensional calculations will be more efficient and accurate than Jameson's dissipation model, if multigrid with Runge-Kutta timestepping and frozen dissipation is applied.

\section{References}

1. A. Brandt, Guide to multigrid development, in: Multigrid Methods (W. Hackbusch and U. Trottenberg, eds.), Lecture Notes in Mathematics 960 (1981) 220-312, Springer Verlag, Berlin.

2. R. Courant and K.O. Friedrichs, Supersonic Flow and Shock Waves, Interscience, New York (1967).

3. H. Deconinck, A survey of upwind principles for the multidimensional Euler equations, VKI Lecture Series on Computational Fluid Dynamics 1987-04 (1987).

4. E. Dick, Relaxation of steady flow equations, VKI Lecture Series on Computational Fluid Dynamics 1988-05 (1988).

5. B. Gustafsson and P. Lötstedt, Analysis of the multigrid method applied to first order systems, Report No. 120/1989, Uppsala University (1989). 
6. A. Harten and S. Osher, Uniformly high-order accurate non-oscillatory schemes I, SIAM J. Numer. Anal. 24 (1987) 279-309.

7. A. Harten, B. Engquist, S. Osher and S. Chakravarthy, Uniformly high order accurate essentially nonoscillatory schemes, III, J. Comp. Phys. 71 (1987) 231-303.

8. A. Jameson, Transonic flow calculations, MAE-report 1651, Princeton University (1983).

9. B. Koren, Multigrid and defect correction for the steady Navier-Stokes equations, Ph.D. Thesis (1987).

10. M.H. Lallemand and B. Koren, Iterative defect correction and multigrid accelerated explicit time stepping schemes for the steady Euler equations, Centre for Mathematics and Computer Science, Amsterdam (1989).

11. S. Osher and S. Chakravarthy, Very high order accurate TVD schemes, The IMA Volumes in Mathematics and its Applications 2 (1986) 229-274, Springer Verlag, Berlin.

12. P.L. Roe, Approximate Riemann solvers, parameter vectors and difference schemes, J. Comp. Phys. 43 (1981) 357-372.

13. S.P. Spekreijse, Multigrid solution of the steady Euler equations, Ph.D. Thesis (1989).

14. K. Stuben and U. Trottenberg, Multigrid methods: Fundamental algorithms, model problem analysis and applications, in: Multigrid Methods (W. Hackbusch and U. Trottenberg, eds.), Lecture Notes in Mathematics 960 (1981) 1-176, Springer Verlag, Berlin.

15. H.C. Yee, Upwind and symmetric shock capturing schemes, NASA Technical Memorandum 89464 (1987). 